\title{
4Pi microscopy of quantum dot-labeled cellular structures
}

\author{
Rebecca Medda, Stefan Jakobs, Stefan W. Hell, Jörg Bewersdorf* \\ Max Planck Institute for Biophysical Chemistry, Department of NanoBiophotonics, 37070 Göttingen, Germany
}

Received 8 April 2006; received in revised form 18 August 2006; accepted 19 August 2006

Available online 7 September 2006

\begin{abstract}
The most prominent restrictions of fluorescence microscopy are the limited resolution and the finite signal. Established conventional, confocal, and multiphoton microscopes resolve at best $\sim 200 \mathrm{~nm}$ in the focal plane and only $\geqslant 500 \mathrm{~nm}$ in depth. Additionally, organic fluorophores and fluorescent proteins are bleached after $10^{4}-10^{5}$ excitation cycles. To overcome these restrictions, we synergistically combine the 3- to 7-fold improved axial resolution of 4Pi microscopy with the greatly enhanced photostability of semiconductor quantum dots. Co-localization studies of immunolabeled microtubules and mitochondria demonstrate the feasibility of this approach for routine biological measurements. In particular, we visualize the three-dimensional entanglement of the two networks with unprecedented detail. (C) 2006 Elsevier Inc. All rights reserved.
\end{abstract}

Keywords: Confocal; Multiphoton; Two-photon; Interference; QDot; Fluorescence; Mitochondria; Microtubule; PtK2

\section{Introduction}

The quest to explore the spatial distribution of proteins in entire cells in three dimensions (3D) is severely hampered by the resolution bounds of established forms of fluorescence microscopy. Even for the lenses of the highest achievable numerical aperture (NA), the currently available 3D-resolution of confocal microscopy is limited by diffraction to $>200 \mathrm{~nm}$ in the focal plane and $\geqslant 500 \mathrm{~nm}$ along the optic axis (Pawley, 2006). By employing two high-NA lenses in a coherent manner (their light fields are added rather than the light intensity), 4Pi microscopy relaxes these restrictions significantly (Hell and Stelzer, 1992a; Hell, 1990). The doubling of the illumination aperture sharpens the effective point-spread function (E-PSF) along the optic axis 3- to 4-fold. In 4Pi microscopy of type $\mathrm{C}$, the same aperture enlargement is implemented in the fluorescence detection, which narrows the main diffraction maximum further by another $30-40 \%$ and improves the axial resolution down to $\sim 100 \mathrm{~nm}$ (Gugel et al., 2004; Hell

\footnotetext{
* Corresponding author. Present address: The Jackson Laboratory, 600 Main St., Bar Harbor, ME 04609, USA; Fax: +1 2072886741.

E-mail address: Joerg.Bewersdorf@jax.org (J. Bewersdorf).
}

and Stelzer, 1992a). Additional improvement is possible by applying image restoration techniques (Hell et al., 1997; Nagorni and Hell, 1998; Nagorni and Hell, 2001a; Nagorni and Hell, 2001b).

A downside of 4Pi microscopy is that the main maximum of the E-PSF is accompanied by two axial lobes stemming from the fact that the two lenses cannot provide a full spherical solid angle. The axial lobes are suppressed most efficiently by a combination of: (i) multiphoton excitation, (ii) confocalization, and (iii) a significant disparity between the wavelengths of excitation and emission (in type $\mathrm{C}$ mode) (Gugel et al., 2004; Hell and Stelzer, 1992b). In multiphoton excitation, the quadratic or higher dependence of the fluorescence on the illumination intensity (Denk et al., 1990) lowers the contribution of the sidelobes with respect to that of the main maximum. Confocalization is helpful (but not mandatory) because the axially shifted side-maxima are detected less efficiently through the detection pinhole. Finally, the wavelength disparity between the infrared two-photon excitation (2PE) wavelength and the fluorescence wavelength prevents effective detection of the excitation side-maxima by the detection sidelobes. By exploiting all three effects simultaneously, 4Pi microscopy of type C features effective sidelobe heights of well below 30\% 
(Gugel et al., 2004; Hell and Stelzer, 1992b). Thus, the effect of the lobes can be unambiguously removed by a simple linear deconvolution. The application of 4Pi microscopy to modern cell biology has been demonstrated with a $2 \mathrm{PE} 4 \mathrm{Pi}$ setup of type A (featuring detection with a single lens and 4Pi excitation) in several publications (Egner et al., 2002; Egner et al., 2004). The technique has also recently been implemented in a state-of-the-art beam scanning confocal microscope thus making it more accessible to a larger scientific community (Gugel et al., 2004).

Unfortunately, the observation of small fluorescent structures in biological cells is also limited by a finite signal. Small objects can hold or be coupled to similarly small amounts of fluorophores. The usually low total fluorescence thus may conceal the underlying structures. Long signal accumulation times are generally not a solution, as the overall obtainable signal is limited by photobleaching. For example, even under favorable conditions, the fluorescent proteins EGFP and DsRed emit only up to $\sim 100,000$ fluorescence photons in total when excited by one-photon excitation (Heikal et al., 2000; Peterman et al., 1999). Popular organic fluorophores such as Rhodamine $6 \mathrm{G}$ feature similar fluorescence photon yields (Eggeling et al., 2005). With 2PE, the photobleaching probability of a molecule in focus can be significantly larger (Eggeling et al., 2005). Given that only about $1-10 \%$ of the emitted photons are typically detected, the achievable signal is rather limited.

Fortunately, the advent of water-soluble fluorescent semiconductor quantum dots (Bruchez et al., 1998; Chan and Nie, 1998) presents a solution to this problem for those imaging applications in which quantum dot labeling is admissible and viable. Quantum dots are less prone to bleaching as compared to organic fluorophores (Jaiswal et al., 2003; Wu et al., 2003) and, therefore enable much higher signal accumulation. They also possess a sharp emission spectrum $(\sim 25 \mathrm{~nm}$ width) which is particularly useful in multicolor applications. Moreover, with their 2PE action cross-section of up to $\sim 4.7 \times 10^{-46} \mathrm{~cm}^{4}$ s (Larson et al., 2003) which is about 200-fold larger than for commonly used fluorescent proteins and organic fluorophores (Heikal et al., 2001; Heikal et al., 2000; Xu et al., 1996) quantum dots are well excitable in the 2PE mode. A sub-mW laser power suffices to almost saturate their fluorescence emission. Hence, the 4Pi microscopy conditions (i) and (ii) can be perfectly fulfilled by the use of quantum dots, thus resulting in a nearly perfect synergy of the two techniques.

Due to their rather broad 2PE spectra, quantum dots also facilitate the simultaneous excitation of multiple colors which enables the implementation of large wavelength disparities. As an important side benefit, the broad 2PE excitation spectrum of quantum dots also allows simultaneous multicolor detection with a single $2 \mathrm{PE}$ wavelength. While the synergy of the two imaging techniques is certainly appealing from the optical point-of-view, its usefulness in biology also depends on several other factors, such as the labeling chemistry. Quantum dots with bio-functionalized surfaces, including those conjugated with antibodies (ABs), are now commercially available. However, their comparatively large diameter (>10 nm, including conjugated $\mathrm{ABs}$ ) to some extent questions their applicability in immunolabeling sub-cellular compartments. In this paper, we demonstrate the synergistic application of quantum dot labeling in the most sophisticated and powerful version of $4 \mathrm{Pi}$ microscopy of type C. While two-color 4Pi microscopy of type A had already been realized (Kano et al., 2002), this work illustrates that two-color 4Pi imaging of type $\mathrm{C}$ is feasible. In addition we show as an exemplary study that the resulting data enables the analysis of the intimate spatial mingling of mitochondria with the microtubular network in mammalian PtK2 cells.

\section{Material and methods}

\subsection{Cell culture and immunocytochemistry}

PtK2 cells were grown as described previously (Osborn et al., 1977). For immunocytochemistry, the cells were seeded either on standard glass or fused silica coverslips to a confluency of $50-80 \%$ and fixed in $3.7 \%$ formaldehyde for $10 \mathrm{~min}$ at room temperature; fixation was followed by three PBS washes. Subsequently the cells were permeabilized with $1 \%$ Triton X-100 in PBS for 5 min at room temperature. Cells were subsequently washed three times in PBS with 1\% BSA (blocking buffer) and incubated simultaneously with two primary antibodies in blocking buffer (anti- $\alpha$-Tubulin rabbit IgG, final concentration $1 \mu \mathrm{g} / \mathrm{ml}$, Abcam, Cambridge, UK; anti- $\alpha$-subunit of $\mathrm{F}_{1} \mathrm{~F}_{0}$-ATP-synthase mouse $\mathrm{IgG}$, final concentration $1 \mu \mathrm{g} / \mathrm{ml}$, Molecular Probes, Carlsbad, CA). After $1 \mathrm{~h}$ of incubation, the cells were washed three times with blocking buffer and incubated simultaneously with two secondary antibodies (QDot 605 goat anti-rabbit IgG conjugate; QDot 655 goat antimouse IgG conjugate, final concentration $20 \mathrm{nM}$, Quantum Dot Corporation, Hayward, CA) for $1 \mathrm{~h}$. As a control for quantum dot labeling, Oregon Green conjugated secondary antibodies were employed (Oregon Green goat anti-mouse or goat anti-rabbit, $20 \mu \mathrm{g} / \mathrm{ml}$ Molecular Probes, Carlsbad, CA). For imaging, the cells were mounted in $130 \mathrm{mM}$ Tris- $\mathrm{HCl}$ with $87 \%$ glycerol (v/v), pH 7.5, and covered with a second coverslip. Finally, the two coverslips were sealed with silicon glue (Twinsil, Picodent, Wipperfürth, Germany).

\subsection{Confocal microscopy}

For regular confocal microscopy, a TCS SP2 (Leica Microsystems CMS GmbH, Germany) with a $1.2 \mathrm{NA}$ water immersion lens was used (Leica HCX PL APO $63 \times / 1.2 \mathrm{~W}$ CORR). The fluorescence was excited by the $488 \mathrm{~nm}$ laser line of the built-in Argon ion laser via the DD 488/543 excitation beam splitter and detected by one of the photomultipliers of the spectral detector. The detection wavelength range was set to $500-550 \mathrm{~nm}$ for Oregon Green. For the quantum dots, a $20 \mathrm{~nm}$ bandwidth centered at the specified 
emission maximum was chosen. The pinhole was set to one 'Airy unit'. Stacks of $x y$ images consisting of $1024 \times 1024$ pixels of $58 \times 58 \mathrm{~nm}$ size were recorded at $400 \mathrm{~Hz}$ line scan speed. Each line was averaged fourfold. Furthermore, four frames were added for each $x y$ image. The axial distance between adjacent slides was $163 \mathrm{~nm}$, i.e., about a third of the axial FWHM of the confocal spot. The $x y$ images of each data stack were added up to create a $z$-projection. For comparison with live EGFP and DsRed transfected cells, the microscope was used in the sequential scan mode with the same settings as stated above. The Helium Neon laser line of $543 \mathrm{~nm}$ was used for DsRed excitation; the detection range of the second photomultiplier was set to $560-650 \mathrm{~nm}$.

\subsection{Pi microscopy}

A TCS 4PI microscope (Leica Microsystems CMS GmbH, Germany) was used with $100 \times / 1.35$ NA glycerol immersion objective lenses (Leica HCX PL APO 100×/1.35 GLYC CORR). 2PE was accomplished with a modelocked Titanium:Sapphire laser (Mira 900, Coherent Inc., Santa Clara, CA) at $870 \mathrm{~nm}$ wavelength. The fluorescence signal was recorded with two avalanche photodiodes (APDs) connected to the extension port of the scan head via multimode optical fibers. The fluorescence was split in front of the fibers by a dichroic beamsplitter (RSP 625 DCLP, Chroma Technology Corp., Rockingham, VT). Bandpass filters (D605/40 $\mathrm{m}$ and D655/40 m, Chroma Technology Corp.) in front of the APDs ensured that only the fluorescence from one type of quantum dots was recorded with each detector. An additional short pass filter (E750SP, Chroma Technology Corp.) in front of the beam splitter blocked remaining infrared laser light.

The 4Pi module of the TCS 4PI was modified for type C 4Pi microscopy (Gugel et al., 2004). A pair of glass wedges, of which one can be shifted relatively to the other, was added to the lower interference arm. To compensate for the medial thickness of the wedge pair, an optical flat of the average wedge pair thickness was added to the upper interference arm (see Gugel et al. for a sketch of the setup and more details). The 4Pi type $\mathrm{C}$ mode simultaneously utilizes the coherent addition of the focused illumination by the two objective lenses, as well as that of the detection of the fluorescence imaged by both lenses. The use of the two synthesized apertures yields the E-PSF with the highest 4Piresolution possible in this system and lowers the axially shifted secondary interference maxima in the E-PSF, thus minimizing sources of artifacts in the resulting images.

For optimal performance of the $4 \mathrm{Pi}$ system, constructive interference in the focal plane has to be realized for the excitation as well as for both detection channels. The wavelength dependence of the interference can be analyzed by shining an ordinary lamp into the camera port of the Leica microscope. The white light, which is reflected at both sides of a mirrored cover slip in the common focal plane of the objective lenses, interferes at the free side of the 4Pi beam splitter cube and is detected via a multimode optical fiber by a spectrometer. The measured interference spectrum provides a measure for the path length difference between the two interference arms as a function of the wavelength. The one-time adjustment is performed by shifting one of the two glass wedges in the lower arm of the 4Pi interference cavity with respect to the other to change the effective thickness of the wedge pair. In combination with a precise adjustment of the optical path length of the two arms using the upper manually adjustable mirror and the piezo-driven mirror in the cavity, constructive interference for all wavelengths can simultaneously be attained in the focal plane. This produces effective 4Pi PSFs with sidelobes well below 30\%.

The 3D data sets were recorded in the $x z y$ mode of the microscope. Unlike in the confocal microscope $x z$ instead of $x y$-slices were recorded. Each $x z$-slice consists of $512 \times 256$ pixels (400 lines/s, bidirectional scan mode) with a pixel size of $46 \times 23 \mathrm{~nm}$ and was averaged 32 -fold in the recording process $(8 \times$ line average, $4 \times$ frame accumulation) before continuing with the next slice. The $y$-distance between neighboring $x z$-slices was $61 \mathrm{~nm}$. The symmetry of the E-PSF of the 4Pi microscope (constructive interference) was adjusted beforehand at an adjacent section of the cell. Photobleaching could only be observed for laser powers far higher than the sub-mW powers employed. In fact, as photobleaching was negligible, saturation effects that increase the relative height of the secondary E-PSF-maxima were observable as a limiting effect. The excitation saturation eventually curbs the fluorescence photon flux and the imaging speed. The pinhole was set to 0.7 Airy units to optimize the E-PSF of the 4Pi microscope.

To suppress high-frequency shot noise, the data of the two channels of the recorded 3D data stacks was smoothed separately in all directions with a Gaussian filter of sub-diffraction size. Afterwards, the stacks were linearly deconvolved with the inverse function of a simple onedimensional comb function featuring five peaks. The height of the peaks was derived from the relative height and axial positions of the most pronounced axial side maxima of the 4Pi E-PSF function (Nagorni and Hell, 1998). Typical values for the five peaks were $0.05,0.30,1,0.30$, and 0.05 reflecting the high PSF quality. Due to the low values of the secondary and tertiary maxima and the high signal, the deconvolution process is very stable. Negative values in the data which arose after deconvolution were $<10 \%$ of the global maximum intensity in the stack and were set to zero. Such negative values in the linearly deconvolved data arise from noise or small local variations in the height of the peaks in the E-PSF. The latter partially originate from small variations of the relative phase of the wavefronts during scanning. For a 3D-representation of our results we used the 'isosurface' function of the 3D-rendering software Amira (version 3.1, TGS Inc., San Diego, CA).

\section{Results and discussion}

To verify the feasibility of quantum dot stainings in immunocytochemistry, we compared quantum dot labeled 
samples with conventional organic fluorophore labelings. We chose $\alpha$-tubulin as a marker of microtubules. Additionally, we labeled ATP-synthase which is located in the mitochondrial inner membrane, thus marking the mitochondrial network. Labeling the $\alpha$-subunit of the ATP-synthase which faces the matrix of the mitochondria requires the quantum dot conjugated secondary $\mathrm{ABs}$ to cross both mitochondrial membranes and is due to the large quantum dot size especially challenging. Cells were fixed, permeabilized and incubated with the primary ABs (anti- $\alpha$-subunit of the $\mathrm{F}_{1} \mathrm{~F}_{0}$-ATP-synthase and anti- $\alpha$-tubulin) under identical conditions before treated with the fluorescent probe-conjugated secondary ABs. Under optimal staining conditions confocal imaging could not reveal any structural differences between the quantum dot and the organic fluorophore stainings (see Fig. 1). The comparison of the immuno-labeled cells with live cells expressing DsRed in the mitochondrial matrix as well as $\alpha$-tubulin-EGFP, also featured no detectable discrepancy in the observed structures (data not shown). These findings indicate that imaging of the immuno-labeled PtK2 cells largely reflect their in vivo state.

Quantum dot-labeled cells prepared in this way were subsequently recorded with $4 \mathrm{Pi}$ microscopy. Fig. 2A displays the axial projection of a typical $3 \mathrm{D}$ data stack recorded with the $4 \mathrm{Pi}$ microscope of type $\mathrm{C}$. Revealing the mitochondrial network of a single PtK2 cell, the ATP-synthase was immuno-labeled by quantum dots emitting at $655 \mathrm{~nm}$. Fig. 2B and C highlight typical $x z$-slices of the stack. Note the high signal and negligible background of the raw data displayed in the upper frames of Fig. 2B and C. The side-maxima of $\sim 30 \%$ can be easily removed by a simple 5-point deconvolution (Fig. 2B and C lower frames and Fig. 2D and E) (Nagorni and Hell, 1998). Photobleaching was negligible after recording of the 687 frame data set. Even imaging a single $x z$-slice 100 times could not produce detectable photo-bleaching. Therefore, to further raise the signal the recording time can be increased. The $4 \mathrm{Pi}$ imaging of quantum dot-labeled samples is practically not limited by photobleaching.

By simultaneously imaging quantum dots emitting at $605 \mathrm{~nm}$ (revealing tubulin) and quantum dots emitting at $655 \mathrm{~nm}$ (highlighting ATP-synthase), we demonstrate for the first time the viability of two-color imaging in 4Pi microscopy of type C. By carefully adjusting the glass wedge pair used for dispersion correction in the lower interference arm of the 4Pi microscope as well as by a thorough optimization of the optical path length difference between the two arms of the 4Pi module, constructive interference for both detection channels can simultaneously be achieved. Mounting the sample in $87 \%$ glycerol medium minimizes the variation of the
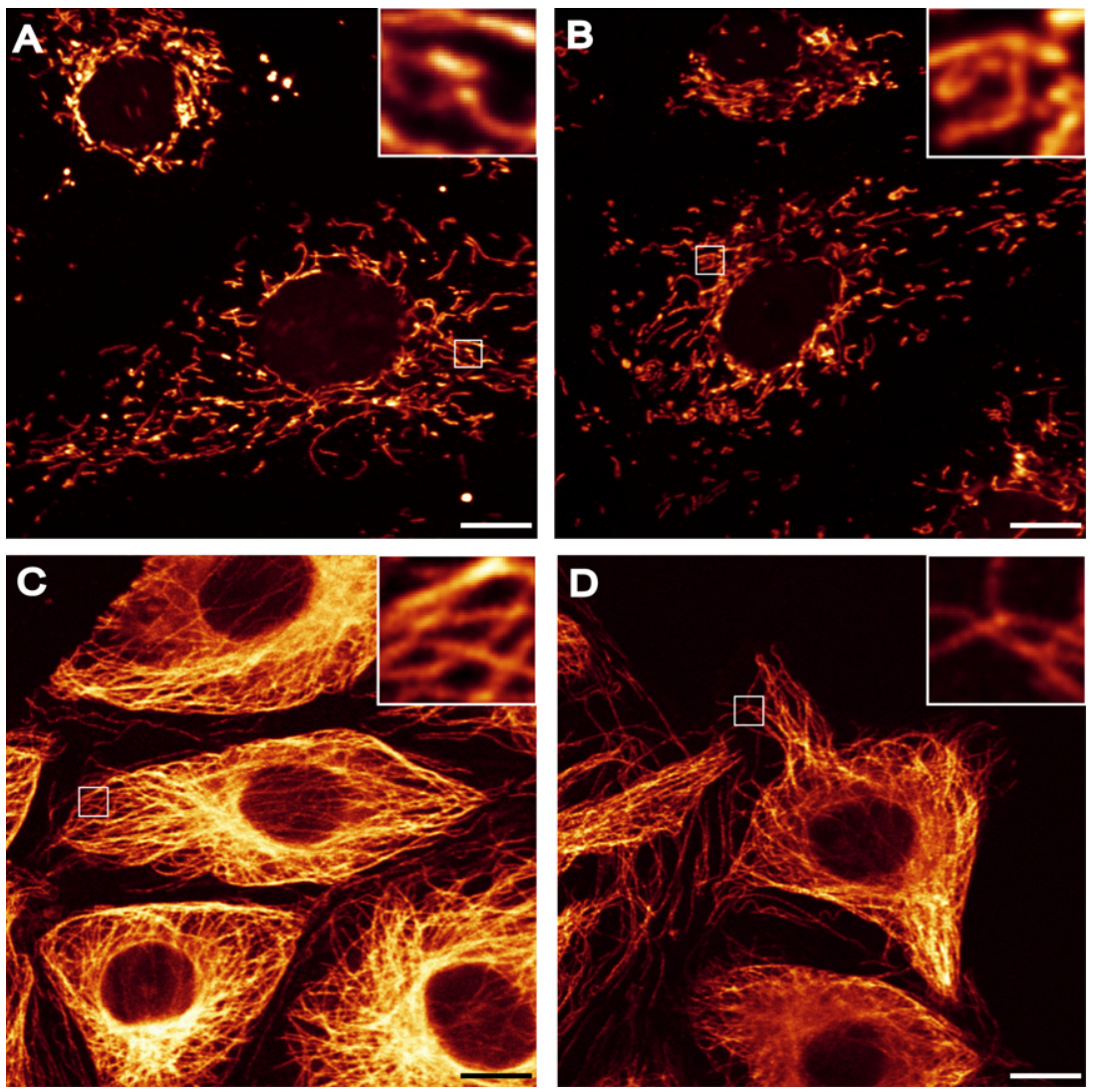

Fig. 1. Comparison of PtK2 cells immunolabeled with Oregon Green and quantum dots. (A and B) Show typical stainings of the $\mathrm{F}_{1} \mathrm{~F}_{0}-\mathrm{ATP}$-synthase located in the mitochondrial inner membrane with Oregon Green and QDot 655, respectively. (C and D) Display the microtubules in PtK2 cells immunolabeled by Oregon Green and QDot 605, respectively. The insets that present enlarged details of the images marked by the white boxes clearly indicate that the quantum dot and Oregon Green stainings produce structurally virtually indistinguishable results. Scale bar: $10 \mu \mathrm{m}$. 

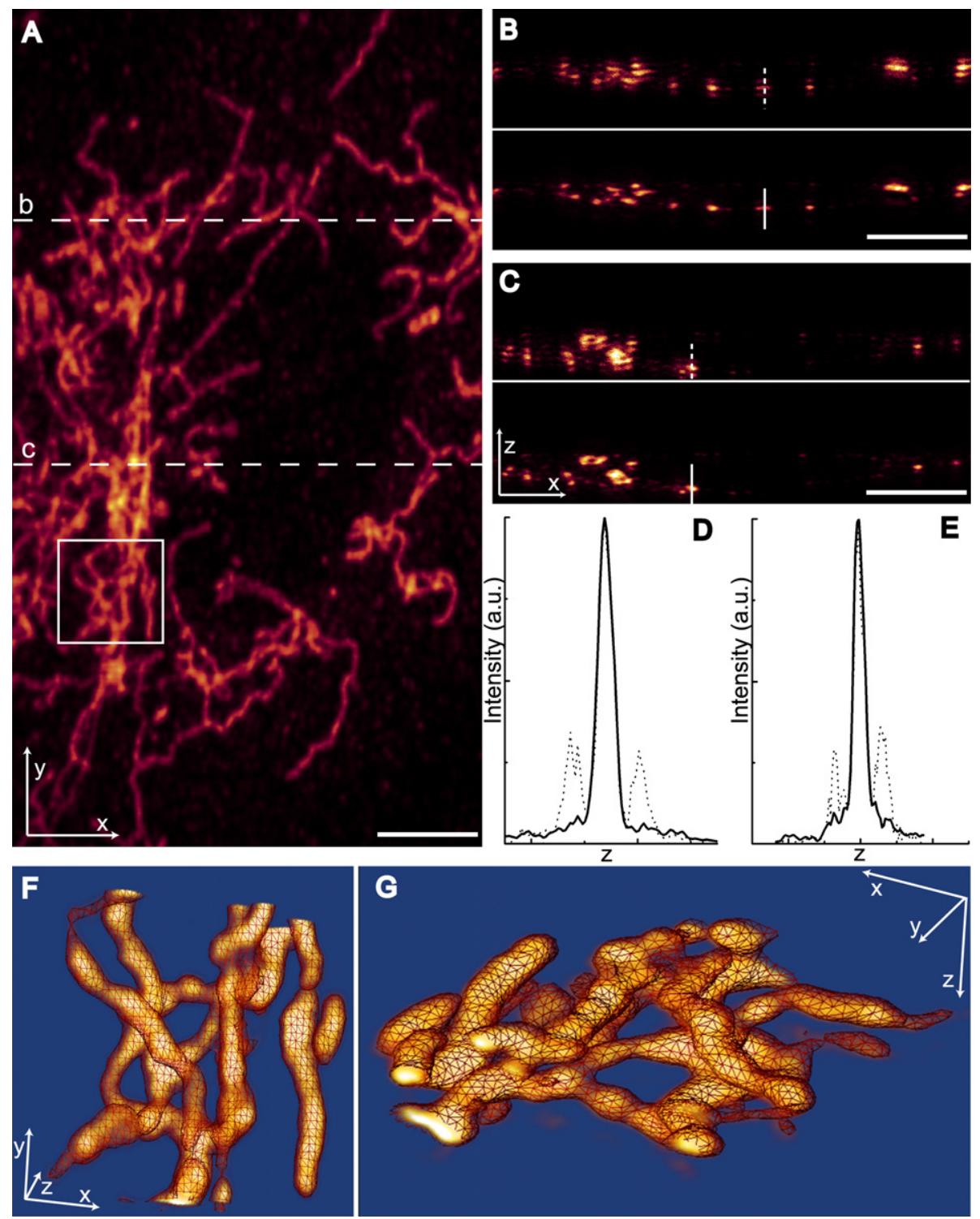

Fig. 2. 4Pi imaging of the mitochondrial structure ( $\alpha$-subunit of the $\mathrm{F}_{1} \mathrm{~F}_{0}$-ATP-synthase) in a PtK2 cell immunolabeled by quantum dots. (A) shows an axial average projection of the recorded 3D data stack. (B and C) Display the $x z$ sections of the 3D data stack marked by the dashed lines (b and c) in (A) (upper image: raw data, lower image: linearly deconvolved). The profiles along the white lines in (B and C) are presented in (D and E), respectively. (F and G) Show 3D representations at different orientations of the volume marked by the white box in (A). Scale bar: (A-C) $5 \mu \mathrm{m}$.

interference conditions in the recording volume. The small bandwidth of the emission spectra of quantum dots leads to very low cross-talk between the two detection channels: for the filters used in our experiments, approximately $4 \%$ and $7 \%$ of the signal detected by the matching detectors is measured by the other detectors for quantum dots emitting at 605 and $655 \mathrm{~nm}$, respectively.

Mitochondria have been known to associate to cytoskeletal elements (Griparic and van der Bliek, 2001). Fig. 3 visualizes the microtubular meshwork (shown in green) that is strongly interwoven with the mitochondrial network (red). Fig. 3A, B, D, and E show 3D-rendered structural details of the complete networks displayed in Fig. 3C. These panels demonstrate the power of $4 \mathrm{Pi}$ microscopy to reveal 3D structural details in the $100 \mathrm{~nm}$ range. These details are barely detectable by other light microscopy techniques, if at all.
For example, Fig. 3B shows an example of a mitochondrial tubule oriented along a microtubule representing the typical relative orientation of the two networks in the periphery of the cell. Fig. 3A and E show ring-shaped mitochondria enclosing microtubules. A mitochondrial tubule accompanied by microtubules on top and beneath is displayed in Fig. 3D. These detailed images highlight the importance of increased axial resolution for high-resolution imaging of complex objects in 3D. The about 6-fold resolution enhancement made possible by $4 \mathrm{Pi}$ microscopy reveals much more details in the $z$-direction, and also discloses small structures in the focal plane that are normally concealed by axially blurred objects that happen to be located above or beneath the focal plane.

The shown data demonstrates the level of detail that can be attained by the combination of $4 \mathrm{Pi}$ microscopy and 

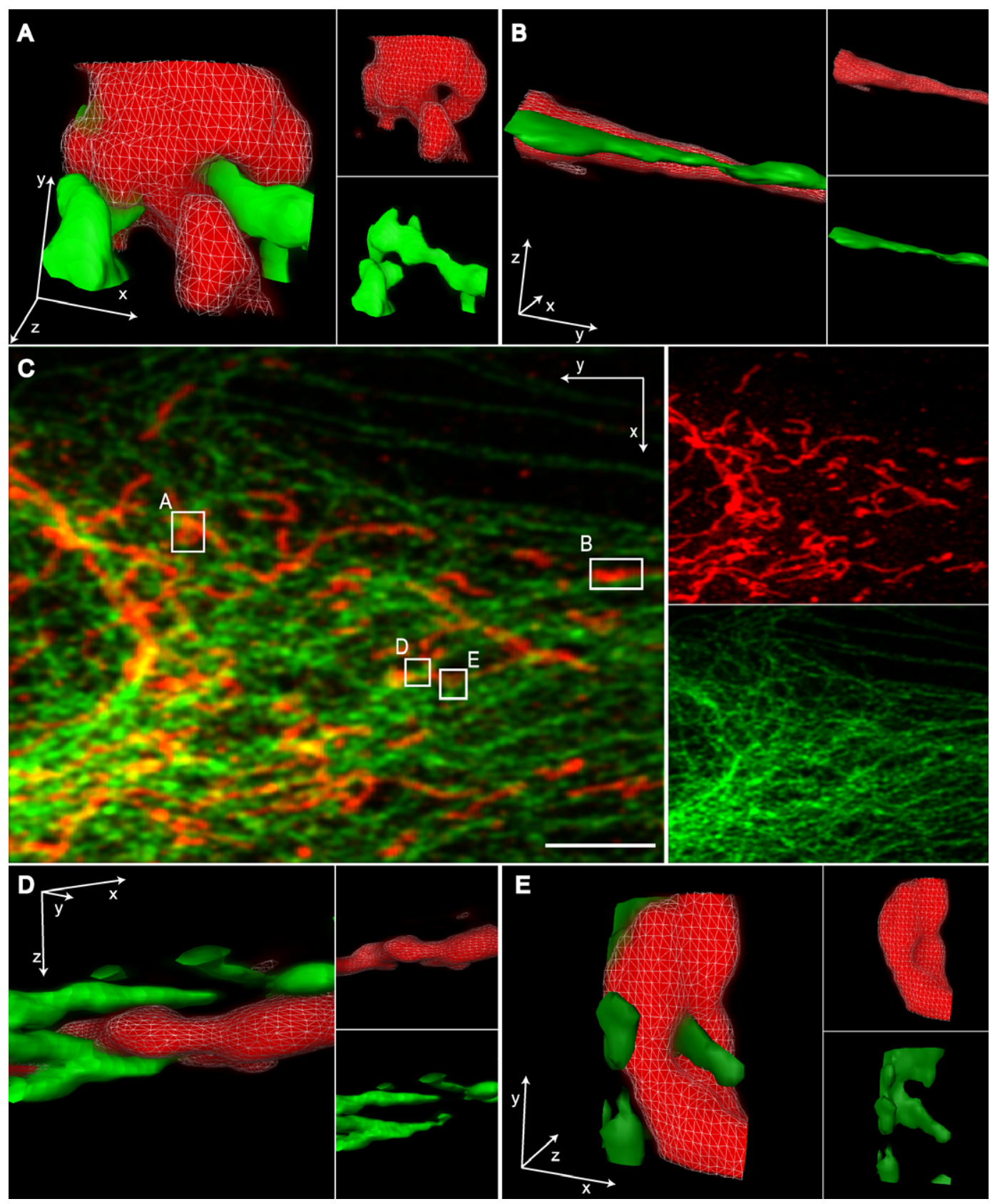

Fig. 3. Two-color 4Pi microscopy with quantum dot labeling: mitochondria ( $\alpha$-subunit of the $\mathrm{F}_{1} \mathrm{~F}_{0}$-ATP-synthase, QDot 655, red) and microtubules ( $\alpha$-Tubulin, QDot 605, green). The central (C) shows the axial average projection of the whole 4Pi data stack; the red and green structures reveal the mitochondria and the microtubules, respectively. The volume data in the area marked by the boxes $(\mathrm{A}, \mathrm{B}, \mathrm{D}$, and $\mathrm{E})$ in the greater $(\mathrm{C})$ are enlarged as $3 \mathrm{D}$ rendered structures to show the amount of structural information contained in the corresponding 3D-data subset. Besides the composite image, each panel shows the two-color channels individually. The arrow lengths of the coordinate systems in the 3D projections correspond to $500 \mathrm{~nm}$. Scale bar in (C): $5 \mu \mathrm{m}$. (For interpretation of the references to color in this figure legend, the reader is referred to the web version of this paper.)

quantum dot labeling. The problem of low signal in highresolution microscopy caused by small detection volumes of the E-PSFs can be counteracted by the use of quantum dots that feature negligible photobleaching. The comparatively strong signal enables the observation of small structural details even if they are surrounded by complex and more dominant structures and enables co-localization studies with unprecedented accuracy. It also opens a window to the observation of low protein concentrations, since lower concentrations of fluorescent markers are now detectable.
The combined use of quantum dots and 4Pi microscopy is also well suited for localization studies as the localization accuracy of a particle depends strongly on the achievable signal and the resolution of the microscope (Thompson et al., 2002). With quantum dots, the high localization accuracies down to the $1 \mathrm{~nm}$ level achieved so far by $4 \mathrm{Pi}$ or standing wave microscopy (Albrecht et al., 2002; Schmidt et al., 2000) get potentially even better. Apart from chromatic aberrations, the larger size of quantum dots compared to organic fluorophores and fluorescent proteins has 
to be taken into account, however, to allow conclusions about the position of the labeled structure from the measured quantum dot position.

Owing to their $\sim 200$-fold increased $2 \mathrm{PE}$ cross-sections as compared to most organic fluorophores, quantum dots allow a significant decrease of the applied laser power while still generating adequate fluorescence. This can be especially important in live cell applications where the avoidance of photodamage is essential. Although most applications of quantum dots have been conducted in conjunction with immunostained fixed cells, recent publications have shown the successful utilization of quantum dots in live cell imaging applications (Alivisatos et al., 2005). By combining this approach with 4Pi microscopy and water immersion lenses (Bahlmann and Hell, 2000), studies with $\sim 100 \mathrm{~nm}$ 3D-resolution in live cells are expected to be possible in the near future.

\section{Acknowledgments}

The authors acknowledge valuable discussions with Andres Kriete, Coriell Institute. We thank Mark Lessard, The Jackson Laboratory, for help with characterizing the quantum dots and Megan McOsker at The Jackson Laboratory for careful reading.

\section{References}

Albrecht, B., Failla, A.V., Schweizer, A., Cremer, C., 2002. Spatially modulated illumination microscopy allows axial distance resolution in the nanometer range. Appl. Optics 41, 80-87.

Alivisatos, A.P., Gu, W., Larabell, C., 2005. Quantum dots as cellular probes. Annu. Rev. Biomed. Eng., 55-76.

Bahlmann, K., Hell, S.W., 2000. Polarization effects in 4Pi confocal microscopy studied with water-immersion lenses. Appl. Optics 39, 1653-1658.

Bruchez Jr., M., Moronne, M., Gin, P., Weiss, S., Alivisatos, A.P., 1998. Semiconductor nanocrystals as fluorescent biological labels. Science 281, 2013-2015

Chan, W.C.W., Nie, S., 1998. Quantum dot bioconjugates for ultrasensitive nonisotopic detections. Science 281, 2016-2018.

Denk, W., Strickler, J.H., Webb, W.W., 1990. Two-photon laser scanning fluorescence microscopy. Science 248, 73-76.

Eggeling, C., Volkmer, A., Seidel, C.A.M., 2005. Molecular photobleaching kinetics of Rhodamine $6 \mathrm{G}$ by one- and two-photon induced confocal fluorescence microscopy. Chem. Phys. Chem. 6, 791-804.

Egner, A., Jakobs, S., Hell, S.W., 2002. Fast 100-nm resolution 3D-microscope reveals structural plasticity of mitochondria in live yeast. Proc. Natl. Acad. Sci. USA 99, 3370-3375.

Egner, A., Verrier, S., Goroshkov, A., Söling, H.-D., Hell, S.W., 2004. 4Pimicroscopy of the Golgi apparatus in live mammalian cells. J. Struct. Biol. 147, 70-76.

Griparic, L., van der Bliek, A.M., 2001. The many shapes of mitochondrial membranes. Traffic 2, 235-244.
Gugel, H., Bewersdorf, J., Jakobs, S., Engelhardt, J., Storz, R., Hell, S.W., 2004. Cooperative $4 \mathrm{Pi}$ excitation and detection yields 7-fold sharper optical sections in live cell microscopy. Biophys. J. 87, 4146-4152.

Heikal, A.A., Hess, S.T., Baird, G.S., Tsien, R.Y., Webb, W.W., 2000 Molecular spectroscopy and dynamics of intrinsically fluorescent proteins: coral red (dsRed) and yellow (Citrine). Proc. Natl. Acad. Sci. USA 97, 11996-12001.

Heikal, A.A., Hess, S.T., Webb, W.W., 2001. Multiphoton molecular spectroscopy and excited-state dynamics of enhanced green fluorescent protein (EGFP): acid-base specificity. Chem. Phys. 274, 37-55.

Hell, S.W., 1990. Double-scanning confocal microscope, European patent 0491289

Hell, S., Stelzer, E.H.K., 1992a. Properties of a 4Pi-confocal fluorescence microscope. J. Opt. Soc. Am. A 9, 2159-2166.

Hell, S.W., Stelzer, E.H.K., 1992b. Fundamental improvement of resolution with a 4Pi-confocal fluorescence microscope using two-photon excitation. Opt. Commun. 93, 277-282.

Hell, S.W., Schrader, M., van der Voort, H.T.M., 1997. Far-field fluorescence microscopy with three-dimensional resolution in the $100 \mathrm{~nm}$ range. J. Microsc. 185, 1-5.

Jaiswal, J.K., Mattoussi, H., Mauro, J.M., Simon, S.M., 2003. Long-term multiple color imaging of live cells using quantum dot bioconjugates. Nat. Biotechnol. 21, 47-51.

Kano, H., Jakobs, S., Nagorni, M., Hell, S.W., 2002. Dual-color 4Pi-confocal microscopy with 3D-resolution in the $100 \mathrm{~nm}$ range. Ultramicroscopy 90, 207-213.

Larson, D.R., Zipfel, W.R., Williams, R.M., Clark, S.W., Bruchez, M.P., Wise, F.W., Webb, W.W., 2003. Water-soluble quantum dots for multiphoton fluorescence imaging in vivo. Science 300, 1434-1436.

Nagorni, M., Hell, S.W., 1998. 4Pi-confocal microscopy provides threedimensional images of the microtubule network with 100 - to $150-\mathrm{nm}$ resolution. J. Struct. Biol. 123, 236-247.

Nagorni, M., Hell, S.W., 2001a. Coherent use of opposing lenses for axial resolution increase in fluorescence microscopy. I. Comparative study of concepts. J. Opt. Soc. Am. A 18, 36-48.

Nagorni, M., Hell, S.W., 2001b. Coherent use of opposing lenses for axial resolution increase in fluorescence microscopy. II. Power and limitation of nonlinear image restoration. J. Opt. Soc. Am. A 18, 49-54.

Osborn, M., Franke, W.W., Weber, K., 1977. Visualization of a system of filaments 7-10 nm thick in cultured-cells of an epithelioid line (Pt K2) by Immunofluorescence Microscopy. Proc. Natl. Acad. Sci. USA 74 2490-2494.

Pawley, J., 2006. Handbook of Biological Confocal Microscopy. Springer, New York.

Peterman, E.J.G., Brasselet, S., Moerner, W.E., 1999. The fluorescence dynamics of single molecules of green fluorescent protein. J. Phys. Chem. A 103, 10553-10560.

Schmidt, M., Nagorni, M., Hell, S.W., 2000. Subresolution axial distance measurements in far-field fluorescence microscopy with precision of 1 nanometer. Rev. Sci. Instrum. 71, 2742-2745.

Thompson, R.E., Larson, D.R., Webb, W.W., 2002. Precise nanometer localization analysis for individual fluorescent probes. Biophys. J. 82, 2775-2783.

Wu, X., Liu, H., Liu, J., Haley, K.N., Treadway, J.A., Larson, J.P., Ge, N., Peale, F., Bruchez, M.P., 2003. Immunofluorescent labeling of cancer marker Her2 and other cellular targets with semiconductor quantum dots. Nat. Biotechnol. 21, 41-46.

Xu, C., Zipfel, W., Shear, J.B., Williams, R.M., Webb, W.W., 1996. Multiphoton fluorescence excitation: new spectral windows for biological nonlinear microscopy. Proc. Natl. Acad. Sci. USA 93, 10763-10768. 